\title{
Autonomous Deployment of a Solar Panel Using Elastic Origami and Distributed Shape-Memory-Polymer Actuators
}

\author{
Tian Chen, ${ }^{1}$ Osama R. Bilal, ${ }^{2}$ Robert Lang, ${ }^{3}$ Chiara Daraio, ${ }^{2, *}$ and Kristina Shea ${ }^{1, \dagger}$ \\ ${ }^{1}$ Engineering Design and Computing Laboratory, Department of Mechanical and Process Engineering, \\ ETH Zurich, Zurich 8092, Switzerland \\ ${ }^{2}$ Division of Engineering and Applied Science, California Institute of Technology, \\ Pasadena, California 91125, USA \\ ${ }^{3}$ Lang Origami, Altadena, California 91001, USA
}

(Received 22 October 2018; revised manuscript received 14 April 2019; published 28 June 2019)

\begin{abstract}
We introduce a metamaterial-based self-deployable system with a rotational periodicity. As a demonstration, we propose an autonomous solar panel array that is programmed to self-deploy in response to changes in the surrounding temperature. We achieve shape reconfiguration and structural stability by exploiting the physical properties in the constituting material and the architecture of the wedge-shaped unit cell. The unit cell consists of one arm of the elastic "flasher" origami and a pair of scissor mechanisms. First, kinematic analysis shows the difference between the theoretical behavior and behavior considering the physical dimensions. This is used to optimize the expansion ratio. Second, the deployment mechanics are enabled through the shape-memory effect inherent in the underlying polymer. A viscoelastic constitutive model is constructed to accurately predict the self-expanding behavior. Lastly, the collapsing and deployment dynamics are discussed. Bifurcation is observed during folding, leading to two different end states, a disk or a cone. By investigating the energy landscape of the system, an apparatus is introduced to enable the disk-shaped folding. A two-stage expansion is observed during deployment. The system first rotates and then expands radially. The resulting system is three-dimensionally (3D) printed, achieves an expansion ratio of $1000 \%$ in under $40 \mathrm{~s}$, and shows excellent agreement with simulation prediction both in the collapsed and expanded configurations.
\end{abstract}

DOI: 10.1103/PhysRevApplied.11.064069

\section{INTRODUCTION}

The surging demand for deployable mechanical systems is predominantly driven by the need to explore ever more inaccessible environments and to deliver increasingly large and complex payloads. Existing needs for deployable systems include space-based solar power [1,2], geoengineering [3], antennas [4], and propulsion [5]. The design of these mechatronic systems focuses on the stowage of the payload [6] and the actuation method that transforms the payload from the stowed to the deployed configuration [7]. Traditionally, actuation is achieved through a network of controlled actuators, which unpacks the passive payload. While numerous studies are carried out to ensure a robust mechanized stowage and deployment process, with

\footnotetext{
*daraio@caltech.edu
}

†kshea@ethz.ch

Published by the American Physical Society under the terms of the Creative Commons Attribution 4.0 International license. Further distribution of this work must maintain attribution to the author(s) and the published article's title, journal citation, and DOI. ever more complex systems, the inherent behaviors of the constituting material become a viable means via which to achieve large-scale autonomous deployment.

Programmable matter is matter that can change its physical properties, e.g., mechanical stiffness, in response to user input or a change in its external environment, e.g., an increase in ambient temperature [8]. Functional materials are types of programmable matter that exhibit these changes through the underlying physical phenomenon, e.g., viscoelasticity and hydrophobic interactions. Numerous designs have begun to utilize such materials to demonstrate functionalities such as shape change [9]. We propose a metamaterial with a rotational periodicity featuring embedded functional materials, i.e., a functional metamaterial, to achieve autonomous deployment of complex systems. Further, we fabricate the metamaterial-based designs using three-dimensional (3D) printing.

Four-dimensional (4D) printing refers to $3 \mathrm{D}$ printing of designs consisting of smart materials that transform over time when exposed to certain stimuli [10]. Existing work primarily demonstrates shape changing behavior. Examples include the fabrication of swelling hydrogel to create doubly curved surfaces [11], programming and activation 
of shape-memory polymers (SMPs) to transform a sheet into a box [12] or to deploy a shape that was stowed in a cylinder [13], and transformable sheets using liquid-crystal elastomers [14] or printing prestrain [15]. Functionalities beyond shape change have also been demonstrated in 4D printed designs. Pneumatics have been used to actuate soft crawlers [16], a robotic octopus [17], and transformable surfaces [18]. Instability has been used to create multistable structures without mechanical hinges [19] and the actuation of soft directional swimmers [20].

Works such as the robotic octopus [17] or the directional swimming robot [20] eliminate the need for a physical tether or onboard electronics and demonstrate a viable means of creating autonomous systems. However, the active components are still treated as separate entities that must be embedded into a passive vessel. This limits the scalability of the system and complicates the design process.

By using advanced fabrication, it becomes possible to embed and distribute sensing, control, and actuation within the programmable matter itself rather than as discrete components in an assembly [21]. In this work, by adopting a functional-metamaterial-based approach, we demonstrate rich autonomous functionalities that originate from simple underlying physical behaviors. Using the example of a self-deploying soft solar-panel array, we aim to demonstrate stable self-configuration through distributed actuation by exploiting the shape-memory effect.

The proposed functional metamaterial consists of $n$ wedge-shaped unit cells, each comprised of an outer ring and an inner substrate [Figs. 1(a) and 1(b)]. The outer ring serves as the primary structure and actuator. The inner substrate carries the solar panels and serves as the secondary actuator. In such a configuration, we show that the inner substrate and the solar panels fit entirely within the inevitable void of the collapsed outer ring. To achieve a tunable large expansion ratio, the outer ring of the unit cell adopts the design of the single-degree-offreedom (single-DOF) Hoberman ${ }^{\mathrm{TM}}$ sphere [22]. The inner

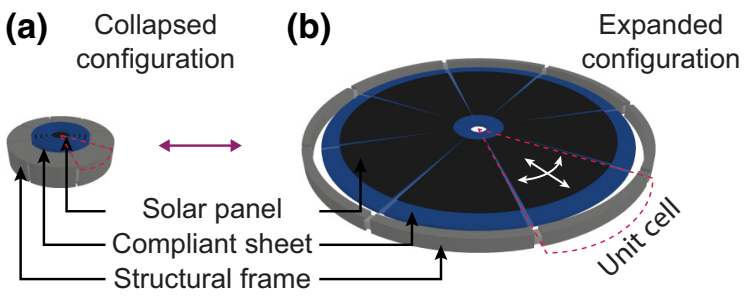

FIG. 1. The schematic of collapsing and deployment from (a) to (b) of the proposed functional metamaterial. An outer ring acts as the primary actuator and structural support. An inner substrate forms the secondary actuator and provides the surface to carry the solar panels. Both components are fabricated using a SMP as the means of actuation. wedge is formed from one section of the "flasher" origami pattern [23].

We pose the maximization of the expansion ratio and the maximization of the number of fitted solar panels as discrete problems that are solved analytically considering kinematics. We demonstrate that the expansion ratio is governed by the periodicity and the radius of the unit cells. The resulting system is able to achieve an area expansion ratio of ten, from approximately 0.05 to $0.5 \mathrm{~m}^{2}$.

The dynamics of folding the "flasher" origami are studied in detail, as a bifurcation behavior is observed in both simulation and experiments. It is shown that in simulation depending on the rate of folding, the origami folds into either a cone or a disk shape. As a result, a rotational center core is devised to collapse the whole system into its stowed configuration.

Both the outer ring and the origami are adapted from pure mechanisms to elastic structures by embedding SMPs in an architected manner. They work in concert to achieve self-deployment when the surrounding temperature increases. Material characterization of the SMP is conducted to determine the triggering temperature, i.e., the glass-transition temperature $T_{g}$, and to propose a linear viscoelastic constitutive model. It is shown that in both stowed and operating configurations where the temperature is lower than the $T_{g}$ of the polymer, both components contribute to the system's overall stiffness. During collapsing and deployment, sensing a temperature higher than $T_{g}$, the material stiffness reduces by several orders of magnitude and becomes compliant. In this example, collapsing to the stowed configuration is manual and programs a prestrain in the SMPs. The autonomous self-deployment occurs when the reduction in stiffness relaxes the prestrain and reconfigures the system.

\section{RESULTS AND DISCUSSION}

The behavior of the self-deploying solar-panel array is described in terms of (1) kinematics, i.e., optimization of the area expansion ratio, (2) materials and mechanics, i.e., the physical properties of the SMP and the mechanics of self-deployment, and (3) dynamics, i.e., analysis of the collapsing and expansion of the array.

\section{A. Optimization of the area expansion ratio}

In existing deployable systems, compliant surfaces such as origami typically serve as passive components to be unfolded with active machineries [5]. Scissor mechanisms have been proposed as a means of deploying passive surfaces [24] (Fig. 2). By embedding SMPs, we enable the self-expansion of such a mechanism. We do not change the underlying kinematics.

We first consider the expansion characteristics of the scissor mechanisms. Following this, the folding constraints 


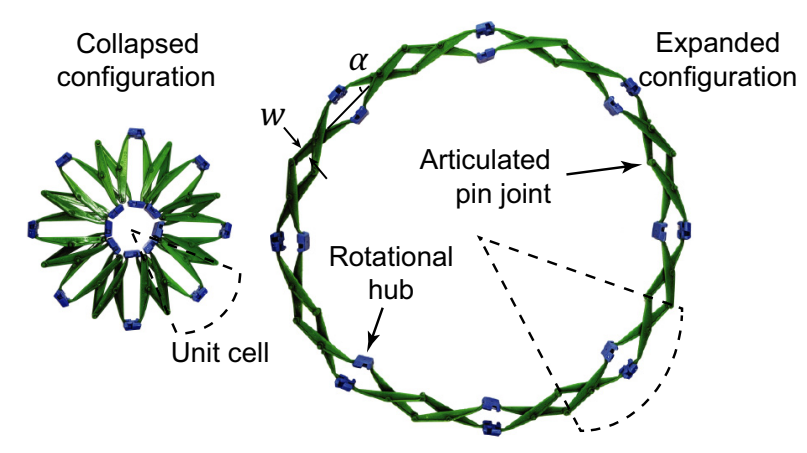

FIG. 2. A ring of self-deploying scissor mechanisms. A ring is extracted from the Hoberman-sphere toy and shown in both collapsed and expanded configurations. The definitions of a unit cell and of the articulated pin joints and rotational hubs are shown.

of the origami substrate are defined. The scissor mechanisms are investigated by characterizing the influence of the rotational periodicity of the unit cell on the expansion ratio, given a fixed radius. The Hoberman-sphere toy makes clever use of the classic scissor mechanisms [25] to create a spherelike object that can expand to several times its collapsed volume. Articulated hubs and pin joints are placed at the intersection of the scissors to enable the mechanism. We adopt a two-dimensional (2D) version (i.e., a ring) of this design (Fig. 2) for the solar-panel array.

The ring is constructed by rotationally tiling $n$ scissor mechanisms around a common center (Fig. 3). This forms a regular polygon as the base geometry of $n$ edges. The expansion ratio of the mechanism is determined by $n$ and by a maximum outer radius limited by the fabrication space. We define the expansion ratio as $\Delta A=$ $A_{\text {expanded }} / A_{\text {collapsed }}$. The maximum and minimum radii, $r_{\max }$ and $r_{\min }$, are measured as the distance between the center and the outermost vertex of the geometry.

The collapsing motion is parametrized by the angle $\alpha$, defined as the angle between the central line connecting two consecutive pins and the mechanism bar [Fig. 3(b)]. The fully expanded configuration is defined by $\alpha=\alpha_{\min }$ and the fully collapsed configuration by $\alpha=\alpha_{\max }$. The maximum physical width of each scissor segment is $w$.

The geometry of the scissor-mechanism unit cell differs from the classical scissor mechanism by lengthening and shortening the arms of the scissors in an alternating pattern ( $l_{1}$ and $l_{2}$ in Fig. 3) using $\xi$ as a control parameter [Eq. (1)]. This relationship is obtained from the derivation in Eq. (A9). Note that the angle $\alpha$ between each pair of bars must remain identical irrespective of the length difference between $l_{1}$ and $l_{2}$ and over the entire range $\left[\alpha_{\min }, \alpha_{\max }\right]$, to ensure kinematic compatibility, and that $\alpha_{\min }$ must be greater than 0 :

$$
l_{1}=\frac{\xi}{1-(\xi-1) \cos (\pi / n)} l_{2} .
$$

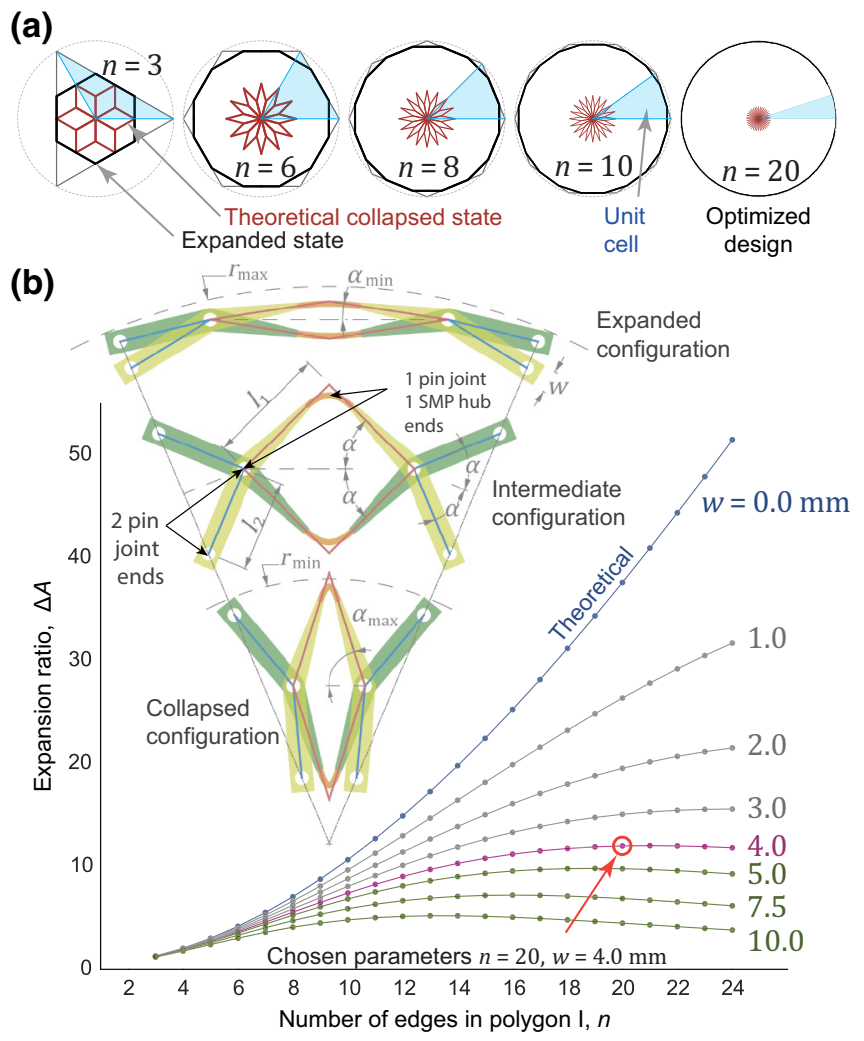

FIG. 3. Parametric optimization of the scissor mechanisms accounting for physical dimensions. (a) Theoretical collapsed and expanded configurations of selected mechanisms. (b) Given a fixed maximum outer radius of $195 \mathrm{~mm}$ as dictated by the fabrication method, the expansion ratio as a function of $n$ is plotted for different member thicknesses, $w$. For member thickness $w=4$, the optimal expansion ratio occurs at $n=20$. The derivation is detailed in Appendix A.

This asymmetry in length accounts for the physical length difference between scissors with one SMP hub end $\left(l_{1}\right)$ and scissors with double-pin-joint ends $\left(l_{2}\right)$. The value of $\xi$ is defined such that in the collapsed configuration, the enveloping radius of the geometry lies on the same circle with a radius $r_{\min }$.

To maximize this expansion ratio, we derive the relationship between $\Delta A$ and the number of polygon sides $(n)$. The schematics (top of Fig. 3) show the geometric configuration of collapsed and expanded rings for different values of $n$, assuming zero thickness, $w$, of the members. In this theoretical scenario, the inner vertices of the ring collapse toward the center of the polygon [26,27]. The derivation of $\triangle A$ considering these parameters is detailed in Appendix A. The resulting maximum angle $\alpha_{\max }$ and the expansion ratio are shown in Eqs. (A15) and (A20). Curves of $\Delta A$ vs $n$ for different values of $w$ are shown in Fig. 3(b).

Qualitatively, it is observed that for a given $w>0$ with increasing $n$, the expansion ratio reaches a maximum $\Delta A$ (Fig. 3). This maximum occurs with smaller $n$ for larger values of $w$, as the thickness of the members prevents the 
mechanism from collapsing to the center. Accounting for fabrication limitations and robustness to repeated cycles of folding and unfolding, we choose a thickness of $w=$ $4 \mathrm{~mm}$ for the experimental realization. For this thickness, the maximum expansion ratio of $\Delta A=11.99$ occurs at $n=20$. The diameter of the inner ring $r_{\text {inner }}$ is used as a constraint for the optimization of the origami substrate:

$$
r_{\text {inner }}=\frac{1}{2} w \cot \frac{\pi}{2 n} .
$$

Origamis consist of a network of crease lines on a substrate that is often inextensional. The crease lines divide the substrate into a number of facets. During folding, the crease lines are assigned either a positive or a negative dihedral angle, resulting in mountain or valley folds. Rigidly foldable origami refer to crease-line patterns that can be folded without inducing strain on the facets.

Origami structures have been studied extensively in the context of deployable systems due to the large difference between the folded and the unfolded configurations [28]. Typically, rigidly foldable origami are used in deployable systems, as their folding kinematics can be predicted with precision [29]. With the introduction of 4D printing, crease lines printed using a SMP within a printed origami pattern can be designed to self-fold [30]. However, as the crease lines must be manually programmed by pulling while under elevated temperature, it seems infeasible to program complex patterns, especially if repeatability and precision are sought. We solve this problem by proposing an elastic origami that can be folded with a single rotation, thereby inducing strain in all crease lines as well as in the facets.

To create a foldable panel with a large expansion ratio and a systematic collapse procedure, we adopt an origami pattern called the "flasher" (Fig. 4) [23]. The "flasher" origami starts as a flat circular disk and can then be folded into a cylinder by allowing each unit cell to fold around the

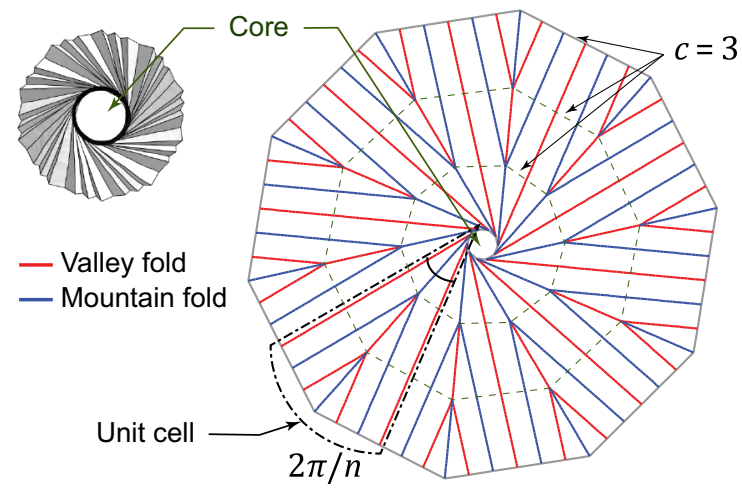

FIG. 4. An elastic "flasher" origami in both collapsed and expanded configurations, with the crease pattern indicated. A unit cell of the origami is defined by the angle of rotation $2 \pi / n$. center core. The wedge-shaped arms of the origami pattern constitute the second part of the unit cell in the functional metamaterial.

The classic "flasher" origami pattern is typically characterized with two variables. The first is the rotational order of the "flasher," $n$, which is also the number of unit cells around the center. The second is the number of circumferential layers in the crease pattern, $c$. The classic pattern is assumed to have zero thickness and is not rigidly foldable. In the interest of deployability, works have been done to modify the pattern to accommodate the physical thickness of the substrate [31] and to allow rigid folding [32]. This modified pattern curves the radial crease lines and introduces a radial cut that must be joined post folding.

By allowing the origami substrate to elastically deform, we circumvent the above modifications to the pattern. The radial crease lines within each unit cell remain parallel. The facades in each unit cell then consist of two triangles and 2(c-1) trapezoids. While flexible solar panels can curve, if they are placed across crease lines, this would increase the folding resistance and they would potentially break during folding. Therefore, they are only placed on the facets in between crease lines. The physical fabrication of the substrate is detailed in Fig. 16 (see Appendix B).

As the origami is intended to serve as a platform, we optimize the crease pattern to fit the maximum number of solar panels of a given size. The objective of the discrete optimization problem considered is to maximize the number of rectangular photovoltaic (PV) cells of the same dimension that are able to fit within one unit cell of a given crease pattern, i.e., $n$ and $c$. The first constraint to this problem states that the outer dimension of the unfolded and folded origami must fit within the expanded and collapsed ring of the scissor mechanisms, respectively. While the dimensions of the unfolded origami can be prescribed prior to fabrication, the dimensions of the folded configuration are derived [Eqs. (B1)-(B9)]. The second constraint states that each facet of the crease pattern must be at least as wide as a given solar panel plus the width of the crease lines [Eqs. (B10) and (B14)]. Preference is given to patterns that can construct periodic linkages to the outer ring.

As this is a 2D optimization problem, the landscape of the objective function as well as the constraints are plotted in Fig. 5. The feasible region shows optimized solutions of 60 solar panels at $(n, c)=(7,5),(10,3),(14,1),(14,2)$. As expected, as the number of rotational-orders $n$ increases, more solar panels can be fitted. The constraints, however, dictate that beyond a certain $n$ or $c$, the folded dimension $r_{f, o}$ exceeds that of the inner dimension of the scissor mechanisms, $r_{\text {inner }}$. The adopted solution is a decagon $(n=10)$ with three layers $(c=3)$. We adopt this configuration because $n=10$ is a fraction of the number of scissor mechanisms. In this way, periodic connections can be made between the two. The derivation of the folded dimension of the origami is detailed in Appendix B. 


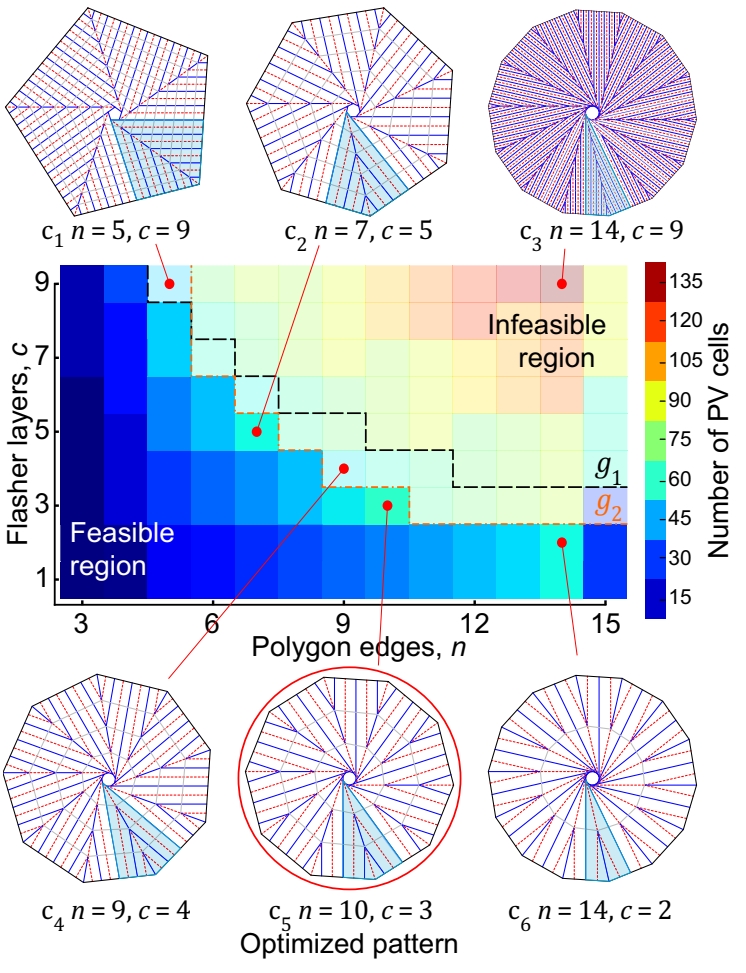

FIG. 5. Maximization of the number of flexible solar panels by changing the "flasher" origami crease pattern. Two variables - $n$, the number of unit cells, and $c$, the number of circumferential layers in the pattern - are optimized to fit the largest number of solar panels of a given dimension. The contour plot shows the number of solar panels that can fit for every pair of $(n, c)$.

\section{B. Mechanics of the shape-memory polymer and of the metamaterial}

Inspired by numerous works that convert pure mechanisms into mechanical metamaterials $[33,34]$, we enable autonomous expansion within the ring by replacing the hubs within the scissor mechanisms with SMP actuators [Fig. 6(a)] and by fabricating the entire origami substrate using the same SMP [Fig. 6(b)]. SMPs are thermosetting polymers that have two distinct mechanical behaviors, depending on their temperature. At a temperature lower than their glass-transition temperature $T_{g}$, the SMPs are in their glassy state, with a high stiffness. When the temperature increases beyond $T_{g}$, they transition to a rubbery state, with a stiffness several orders of magnitude lower than its original value.

Numerous studies have investigated shape-memory effects of 3D-printed polymers [13] as well as their potential applications [20,35]. Autonomous transformation is enabled in a two-stage procedure consisting of programming and recovery. During programming, the SMP is heated past its $T_{g}$ and deformed with the application of a mechanical force. This deformation is maintained as the SMP is cooled to below its $T_{g}$ : this "locks in" the

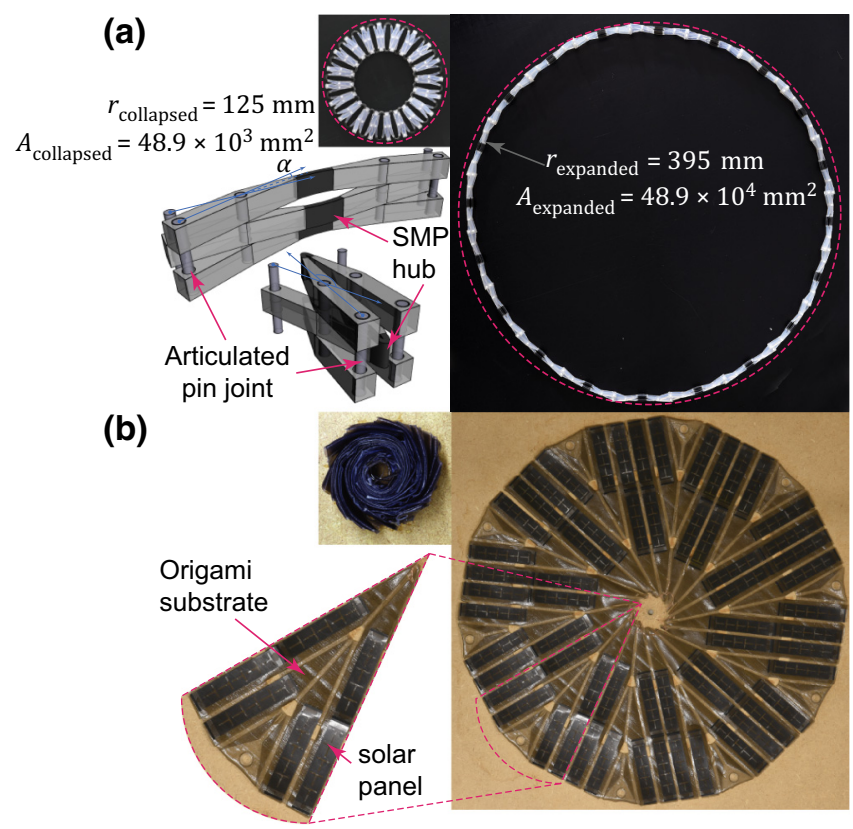

FIG. 6. A fabricated specimen of the scissor mechanisms and of the origami substrate in both collapsed and expanded configurations showing a 10 times change in area. The inset shows one unit cell of the functional metamaterial, consisting of a scissor mechanism and an arm of the origami.

deformation. During recovery, when the SMP is reheated, it autonomously returns to its original configuration and releases the strain energy.

Using differential scanning calorimetry (DSC), a 3Dprinted SMP with a suitable value of $T_{g}$ is selected, with the requirement that the $T_{g}$ of the SMP must be below that of the rigid material used to fabricate the scissor within each scissor mechanism. Then a linear viscoelasticity constitutive model is developed for this 3D-printed SMP using a temperature-controlled frequency sweep with a dynamic mechanical analysis machine. The resulting Prony series is input in a finite-element package for simulation of the shape-memory cycle. This is experimentally validated using a load-testing machine in conjunction with a temperature-controlled chamber. The simulation and experimental procedure are detailed in Appendix C.

As a deploying mechanism that self-expands, we wish to understand the outward pressure that the ring exerts as a function of the angle $\alpha$ between each pair of scissors. The metamaterial approach lends itself neatly to the analysis of the behavior of individual unit cells. In the linear model, the force required to program the SMP is identical to the force that the SMP exerts when it recovers. With simulation, we can obtain an understanding of both the force required to collapse the system and the force that the system exerts during deployment. The unit cell including the SMPs is fabricated in the expanded configuration and programmed to the collapsed configuration. As the Hoberman mechanism has a Poisson's ratio of -1 at all 
strains, the programming is done under $T \geq T_{g}$ by uniformly compressing the ring. This in turn changes the angle $\alpha$ and bends the SMP hub within the scissor mechanisms.

It is known that the recovery force is dependent on the dimensions of the SMP, the strain rate, and the ambient temperature [13]. Our experiments and simulations are performed on one scissor mechanism of the unit cell consisting of one SMP hub under temperature $T>T_{g}$. A prescribed displacement in the compressive direction of $10 \mathrm{~mm}$ is applied to both sides of the scissor mechanism at a rate of $0.5 \mathrm{~mm} \mathrm{~s}^{-1}$. In the experimental setup, the ends of the mechanism are pin connected to the compression plates. The reaction forces are recorded using a load cell with a maximum load of $250 \mathrm{~N}$ and the measurement error is $\pm 0.005 \%( \pm 0.00125 \mathrm{~N})$. Due to the small forces exerted by each shape-memory hub, force-displacement measurements of six specimens are obtained under the same condition. The mean is plotted in Fig. 7(a).

The same geometry is simulated using finite-element analysis. The shape-memory hub is modeled using a linear viscoelastic constitutive model [19] and deformed with a prescribed displacement of $[d, 0,0]$ and $[-d, 0,0]$, where $d=[0-10 \mathrm{~mm}]$ at points $V_{\mathrm{BC}, 1}$ and $V_{\mathrm{BC}, 2}$, respectively. The reaction forces $F_{H}$ are recorded at the same points. The shape-memory hub exhibits a nonlinear forcedisplacement behavior [Fig. 7(a)] as it bends. The numerical and experimental results are comparable, despite the noise introduced due to the low force output. The resulting force $F_{H}$ is plotted against $d$.

The radial pressure $\sigma$ is calculated using trigonometry [Fig. 7(b)]. As $F_{H}$ is horizontal at all stages of loading, the force in the radial direction depends only on the angle $\alpha$. We empirically measure $\alpha$ from the expanded to the collapsed configuration and plot against the radial pressure [Eq. (3)], where $R$ is the outer radius. It is worth noting that the pressure output is proportional to the periodicity of the unit cell:

$$
\sigma=F_{H} \frac{n}{2 \pi R} \frac{\tan (\alpha)}{\cos \left(\frac{\pi}{n}\right)} .
$$

The origami substrate is similarly fabricated using the SMP. It consists of two layers in thickness. The first is a base layer, which is a continuous sheet of SMP $\left(t_{b}\right)$ with voids placed at the center and at the connection points to the scissor mechanism. Additional voids are placed at the intersections of multiple crease lines to reduce stress concentrations. The second is a raised layer consisting of the facets. The thickness of the facets $\left(t_{f}\right)$ provides structural rigidity during the initial folding process but is flexible enough to be wound around the center core. The raised facets confine folding to the regions in between the facets, these gaps forming the crease lines of the origami pattern. As all pairs of facets sharing a crease line always come
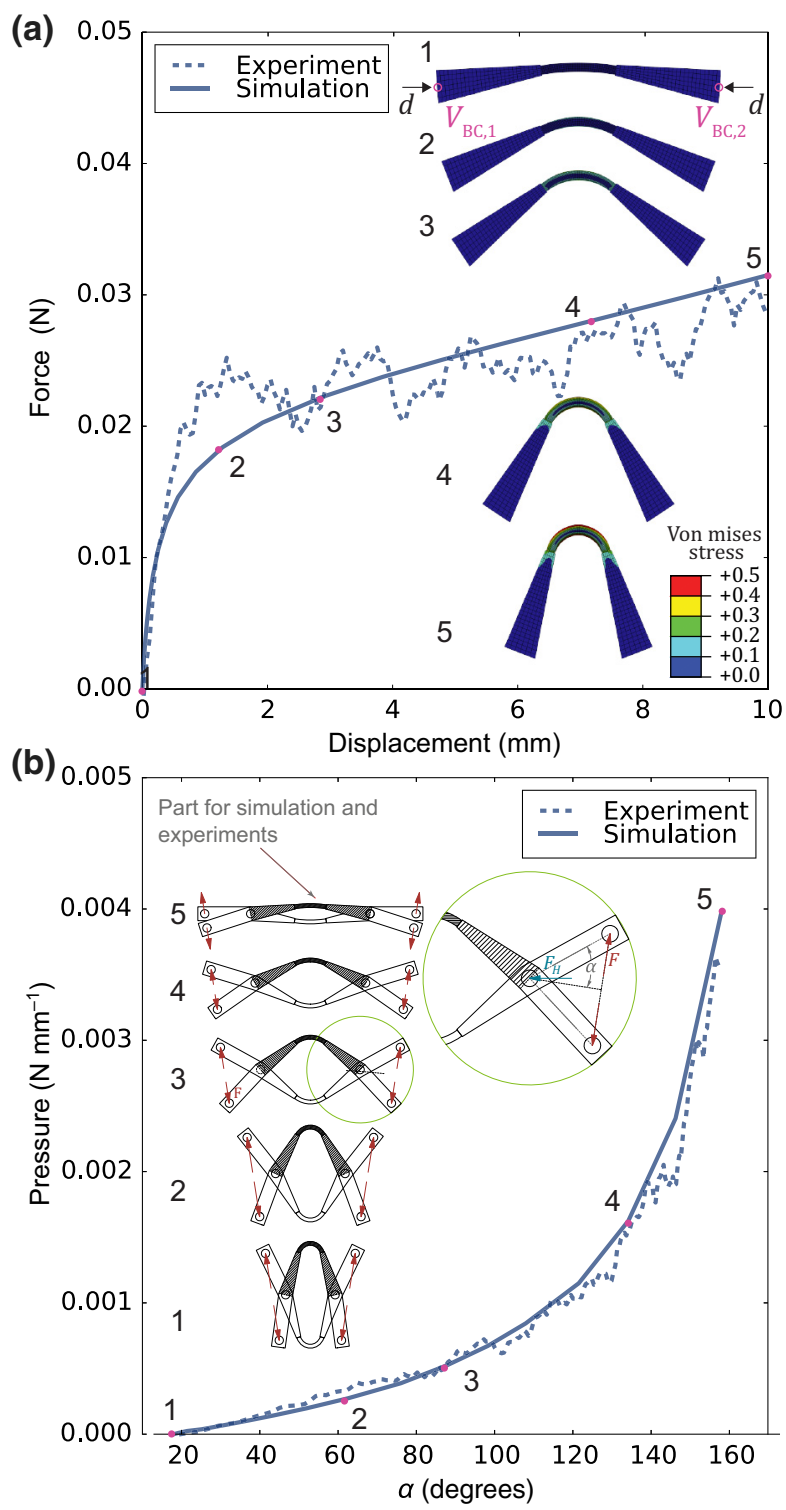

FIG. 7. The mechanics of a scissor mechanism and the relationship between the shape-memory hubs and the radial pressure. (a) A force-displacement plot of a single shape-memory hub from experiments and simulations. (b) The overall pressure produced by all the shape-memory joints of a $n=20$ ring as a function of the angle $\alpha$ in the scissor mechanism.

into contact in the collapsed configuration, i.e., facets that separate two neighboring facets do not exist, the maximum crease-line width $t_{c}$ is twice the facet thickness $2 t_{f}$.

Observing the asymmetric folding behavior (Fig. 8), it is possible to assign a smaller crease-line width to the mountain folds. However, crease lines with a smaller crease line often tear during experiments. As a result, both mountain and valley folds are assigned the same width: $t_{c}=$ $2 t_{f}$. The collapsing procedure of the origami substrate is studied using a simplified model described in the following section. 


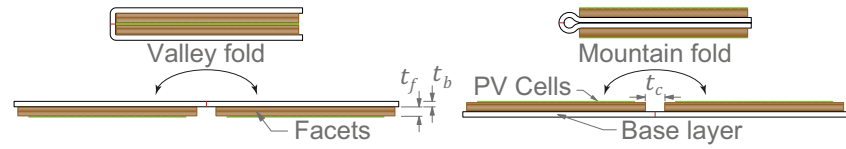

FIG. 8. A schematic of the behavior of mountain and valley folds. The crease-line width $t_{c}$ is dimensioned such that valley folds can be accommodated without inducing unnecessary strain on the base layer.

\section{Dynamics of collapsing and deployment}

To collapse the system, we apply a rotation at the center core while keeping the mountain and valley fold lines of the origami in the correct half plane (i.e., $z>0$ and $z<0$ ) [36]. To apply this rotation, flexible wires are connected between the innermost vertices of the origami and a center rotational core (see Fig. 14). It is noted that during folding, the center may "pop" out of plane, forcing the whole origami to irreversibly assume a conelike shape that needs to be completely flattened prior to refolding. To better understand this phenomenon, the folding process is simulated by assuming the crease pattern as an edge-node network. The coordinates of the nodes are solved such that there is minimal axial strain along the bars and that the dihedral fold angle between all crease lines is equal to a prescribed value $\theta$ [37]. By incrementing $\theta$, a pseudodynamic folding simulation is achieved [38]. As this method is used for rigid origami, the facets of the "flasher" in the tangential direction are finely segmented to mimic an elastic folding behavior. The error is then the elastic energy originating from strain within the bars, as this strain reflects stretching or compression of the panels.

A bifurcation behavior is observed during simulated folding, i.e., two different shapes are achieved from the same fold angles [Figs. $9\left(\mathrm{c}_{1-5}\right)$ and $\left(\mathrm{d}_{1-5}\right)$ show the simulated folding pattern at different fold angles]. Figure 9(a) shows that the height of the cone-shape folding increases linearly until the radial creases are near vertical; whereas for the disk shape, the overall height increases to the width of the facets and the radial creases wind around the center.

In simulation, the cone shape is triggered if the increment in the folding angle $\theta$ is small between two pseudotime-steps and the disk shape is triggered if the increment is large. The strain-energy landscape shows a gradual increase in elastic deformation for the cone-shape folding. However, at a small $\theta$, an abrupt barrier is observed for the disk shape. At larger $\theta$, the disk shape becomes the lower-energy state. This explains the tendency for the center to "pop" out of plane at the start of the folding process. In simulation, larger increment sizes in $\theta$ in effect skip (a)
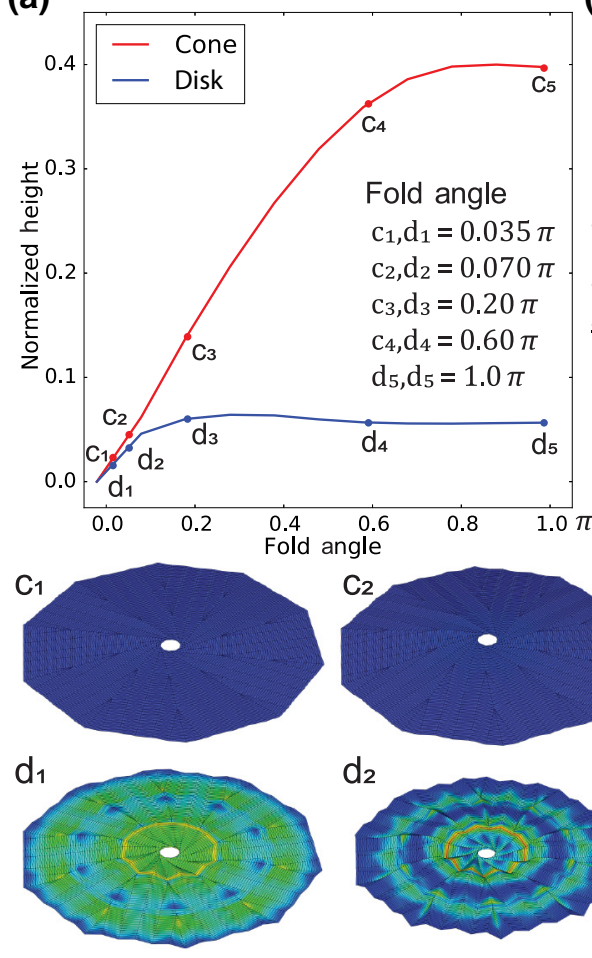

$\mathrm{d}_{2}$ (b)
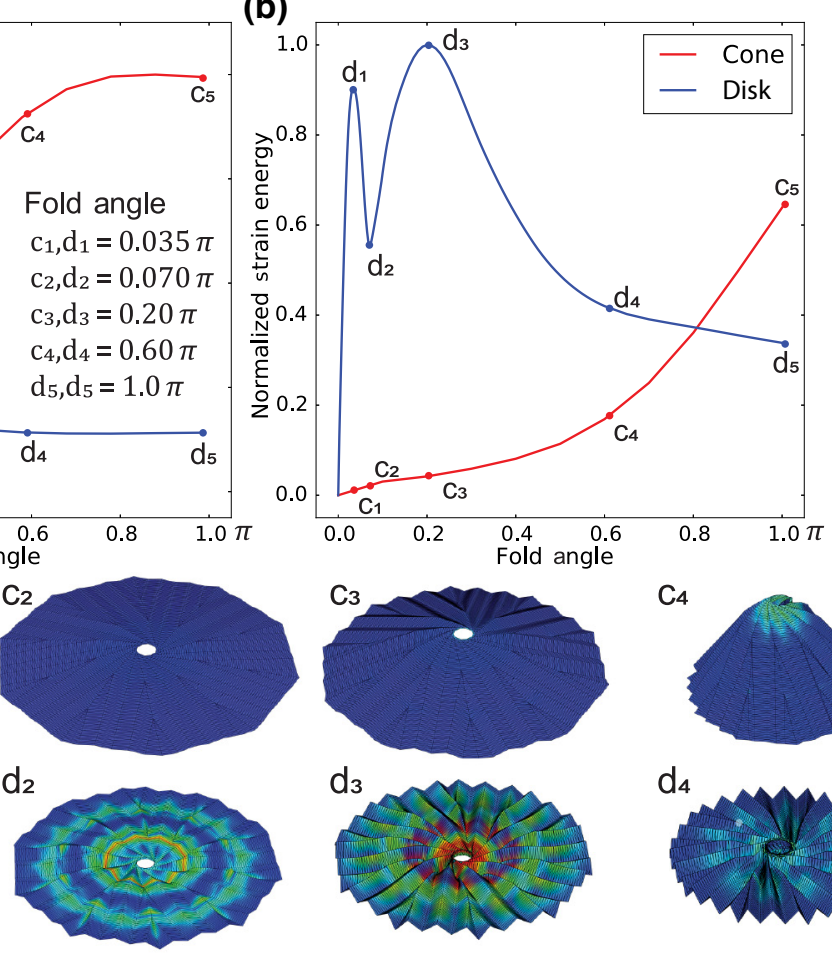
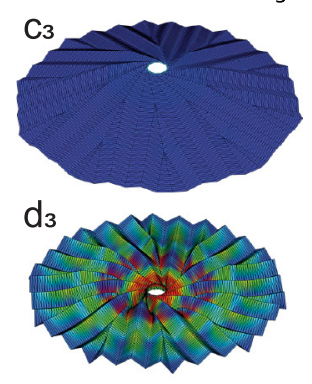

C4

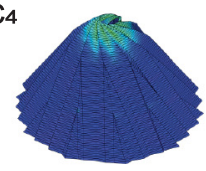

$\mathrm{d}_{4}$

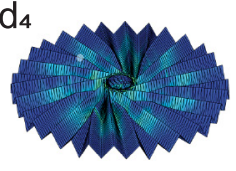

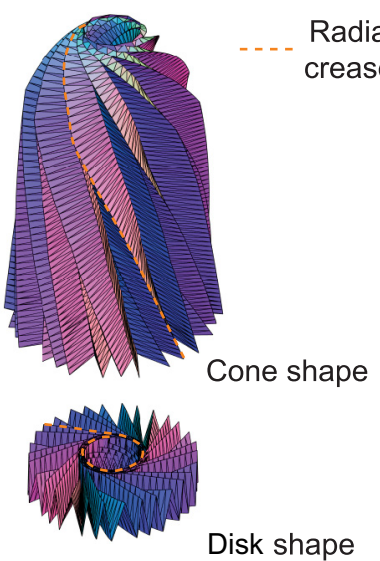

C5

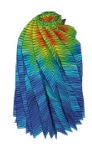

$d_{5}$

FIG. 9. Bifurcation during folding of the "flasher" origami as explained by the elastic energy within the fold lines. Two distinct folded shapes are observed during folding, both in experiment and in simulation. (a) A plot of the total height of the origami as a function of the fold angle (between 0 and $\pi$ ). (b) A plot of the total elastic energy as a function of the fold angle. The resulting folded shapes (i.e., $\alpha=\pi)$ is either a cone or a disk. $\left(c_{1-5}\right)$ and $\left(d_{1-5}\right)$ show different stages of the folding simulations that result in the two eventual shapes. Strain energy is shown as a color gradient. 
(a)

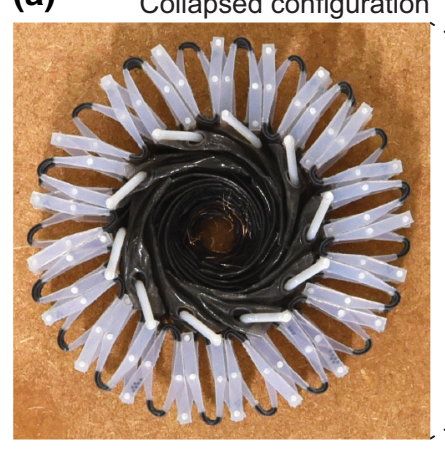

(b)

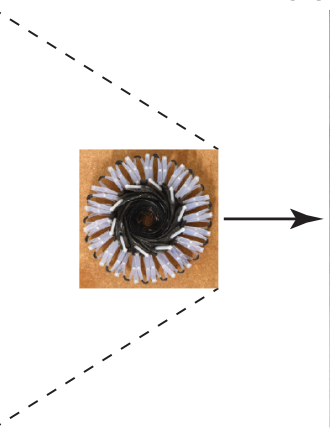

Expanded configuration

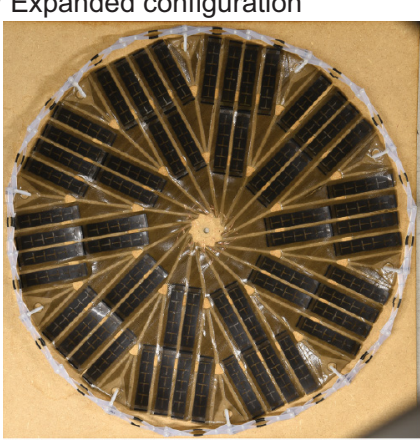

FIG. 10. The deployable solar-panel array. (a),(b) Physical specimens showing the collapsed and the expanded configurations. over this barrier. Experimentally, the same energy barrier is observed during folding. As a solution, the center is manually held down while it is rotated to ensure that the disk shape is achieved.

The metamaterial is assembled by creating a periodic linkage between the origami substrate and the scissor mechanisms. While the system can be physically fabricated using a 3D printer monolithically, the components are fabricated separately and assembled for demonstration purposes. Both the scissor mechanisms and the elastic origami are fabricated in the expanded configuration [Fig. 10(b)]. While both components have been programmed individually for analysis, the assembly is programmed in one step in a heated environment $\left(T \geq T_{g}\right)$.

The collapse starts by rotating the core to which the facets are connected. As the core rotates, the whole geometry is pulled in and wrapped (Video 1). Then a cylindrical mold that would give the desired collapsing ratio is used to demonstrate that the collapsed system can fit within a given space. While confined in this mold, the collapsed system is cooled to the stowing temperature [Fig. 10(a)]. The mold is then removed and the system achieves the stowed configuration.

In contrast to a typical collapsing procedure where the deployable system is a series of mechanisms, the collapsing here is in essence the viscoelastic programming of the shape-memory polymer in the scissor mechanisms
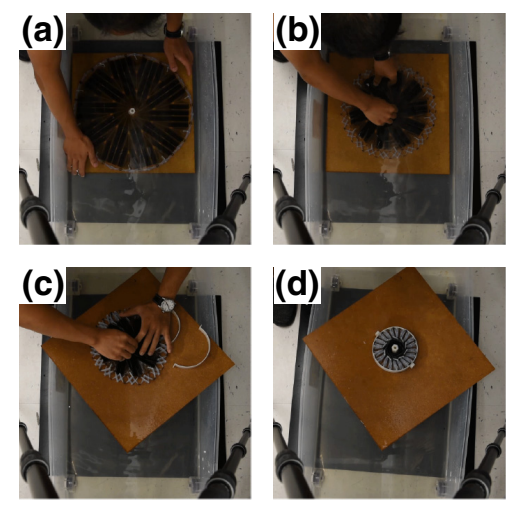

VIDEO 1. The collapsing procedure of the solar-panel array. as well as the origami substrate. The rotational core enables the control of the DOFs that would otherwise immediately relax as the entire metamaterial is in a heated environment.

The system is deployed in an environment heated beyond $T_{g}$ (Video 2). In our experiments, it is deployed in shallow water to simulate a reduced-gravity environment. First, a rapid rotation is observed, the direction being opposite to that of the rotational programming. This rapid motion is largely initiated by the SMP of the origami substrate, which has a higher rate of heat transfer than the SMP hubs due to its large surface area. The rotational motion is caused by the elastic strain encoded in the substrate, both along the panels and across crease lines, during programming. At a certain stage, the rotation stops and the remaining folds are flattened by the scissor mechanisms [Fig. 10(c)]. This deployment behavior mimics a proposed two-stage mechanized deployment of a solar sail [5], yet requires no conventional actuators or power supply.

In a heated environment, deployment will occur regardless of the speed of radial expansion of each component in the functional metamaterial as (1) both components act in an radially outward direction (2) and the two components are physically connected. Qualitatively, this can be explained in the extreme case where one component
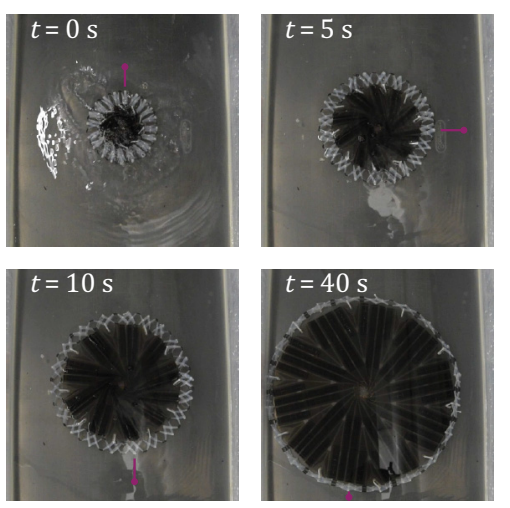

VIDEO 2. The autonomous deployment of the solar-panel array. Video frames of the deployment process in water heated to above $T_{g}$. The dot indicates the relative motion of one unit cell. 
reaches $T_{g}$ much more slowly. Due to the physical connection, if this component remains stiff, the other cannot deploy either (i.e., both programmed strains remain induced). Once the first component eventually reaches $T_{g}$, deployment will occur as the programmed strains in both are relaxed. The rotation that is observed in the first stage of expansion is simply a change in the coordinate system: the scissor mechanism does not experience significant resistance during this rotation, as it is not initially in contact with the surface, i.e., the bottom of the water tank. As the system is under the highest mechanical stress during the collapse process, successful deployment is guaranteed.

\section{CONCLUSION}

We propose an expanding metamaterial with a rotational periodicity and investigate its physical characteristics as well as its applicability to mechanical-engineering applications, e.g., a deployable solar-panel array. Each wedge-shaped unit cell consists of a scissor mechanism as inspired by the Hoberman-sphere toy and an elastic "flasher" origami substrate. We parametrize and optimize the expansion ratio of the scissor mechanism to achieve over $1000 \%$ area change. Using this result as a geometrical constraint, the crease pattern of the "flasher" origami is optimized to accommodate the largest number of solar panels. Second, self-deployment is enabled by embedding a shape-memory polymer (SMP) within each unit cell, both as the rotational hub of the scissor mechanism and as the substrate of the origami. The mechanics of deployment are analyzed by incorporating a linear viscoelastic constitutive model of the SMP in a finite-element package. The force and displacement characteristics are simulated and verified with experiments. Lastly, the dynamics of collapsing and expansion are discussed. A simplified strain-energy model is used to simulate the folding of the origami and to explain the bifurcating behavior exhibited during collapse in experiments. Autonomous deployment with $1000 \%$ area change is achieved in under $40 \mathrm{~s}$. A two-stage expansion behavior is observed: first the system rotates and then it expands radially. This corresponds to the recovery of the origami substrate and of the scissor mechanisms, respectively. We demonstrate the controlled deployment of a mechanical system without conventional actuators, sensors, or a power supply. Rather, it is achieved by embedding and distributing these functionalities within the material and design itself.

\section{ACKNOWLEDGMENTS}

C.D. acknowledges support from the Army Research Office Grant No. W911NF-17-1-0147 and the CaltechJPL President's and Director's Fund Program. The authors thank Jung-Chew Tse for fabrication support. The first author acknowledges fruitful discussions with L. Zimmermann.

\section{APPENDIX A: DERIVATION OF AREA EXPANSION RATIO OF THE RING OF SCISSOR MECHANISMS}

The Hoberman sphere is created using a regular polyhedron as a base. Similarly, the scissor mechanisms created from the Hoberman ring pattern can be created using a regular polygon with $n$ edges. This polygon is referred to as polygon I (Fig. 11). We derive the analytical relationship between the change in area from the expanded to the collapsed configuration of the scissor mechanisms in relation to the number of edges $n$ of the initial polygon. Given the circumscribing radius of the polygon, $r_{\mathrm{OC}, \mathrm{I}}$, as dictated by the maximum fabrication dimension, we calculate the edge length $s^{*}$, and the inscribing radius or the apothem, $r_{\mathrm{IC}, \mathrm{I}}$ :

$$
s^{*}=2 r_{\mathrm{OC}, \mathrm{I}} \sin \left(\frac{\pi}{n}\right) ; \quad r_{\mathrm{IC}, \mathrm{I}}=\frac{1}{2} s^{*} \cot \left(\frac{\pi}{n}\right) .
$$

We introduce an regular polygon with $2 n$ edges, called polygon II, for the construction of the double scissor mechanism. We prescribe that the apothem of this polygon is the same as that of polygon I. We then proceed to derive its side length. From polygon I, we draw a line connecting two

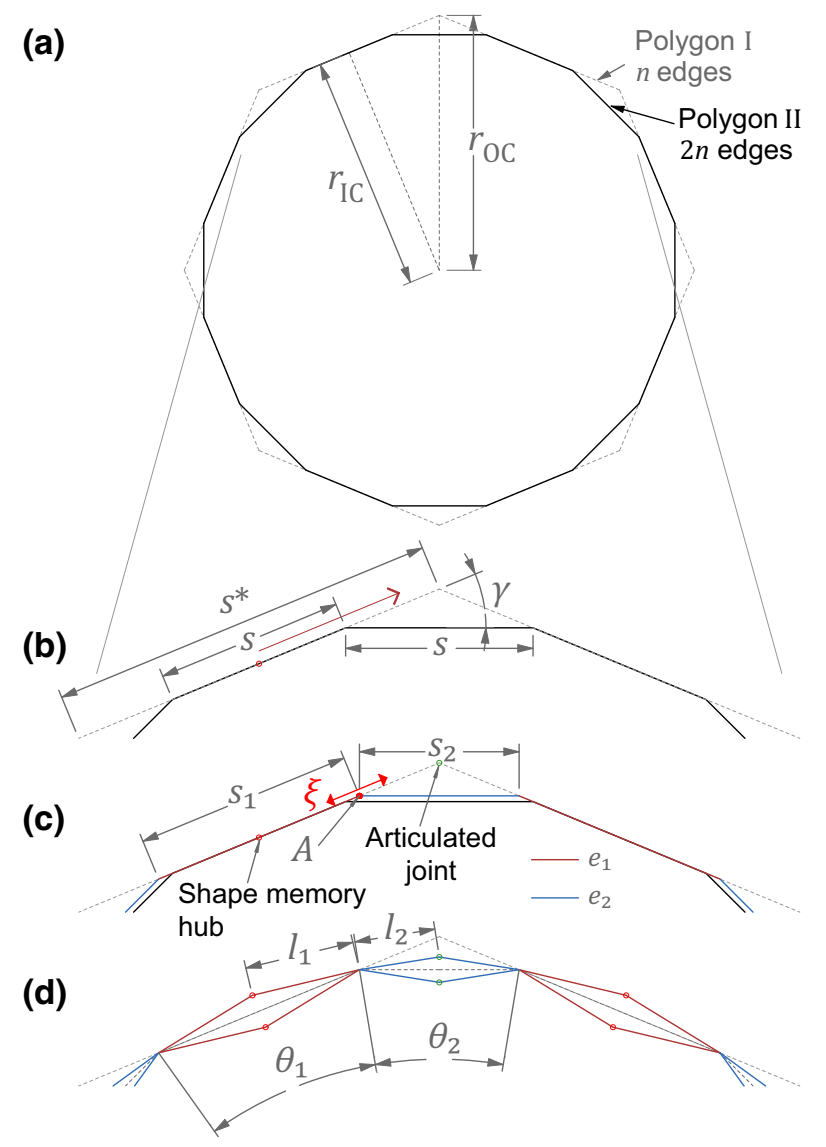

FIG. 11. The construction of the scissor mechanisms. The variables used in the derivation are shown. 
points (e.g., points $A$ and $B$ ) with the same parametrization $t$ on two neighboring edges (the origin being the midpoint of the original edges) to form an isosceles triangle, the two acute angles being equal to $\gamma=\pi / n$ [Fig. 11(b)]. The side length $s$ of polygon II can be derived using the following equation:

$$
\cos (\gamma)=\frac{s}{s^{*}-s}
$$

The solution is as follows:

$$
\begin{aligned}
s & =2 r_{\mathrm{OC}, \mathrm{I}} \cos (\gamma) \frac{\sin (\gamma)}{\cos (\gamma)+1} \\
& =2 r_{\mathrm{OC}, \mathrm{I}} \cos (\gamma) \tan (\gamma / 2) .
\end{aligned}
$$

The parameter $t$ is simply as follows:

$$
\begin{gathered}
t=\frac{s}{s^{*}} \\
=\frac{\cos (\gamma)}{1+\cos (\gamma)} .
\end{gathered}
$$

In the physical design, half the joints are articulated with pins and the other half consist of SMP. It is observed that with polygon II being a regular polygon, the physical edges with pin-joint ends are longer than the ones with SMP ends (Fig. 12). This is due to the added length of the pin-joint ends, as a hole must be made, and to the elastic bending of the SMP. This unnecessarily increases the outer radius of the system in the collapsed configuration.

To address this, the edges of the polygon II are alternatively grouped into two sets, $e_{1}$ and $e_{2}$ [Fig. 11(c)].

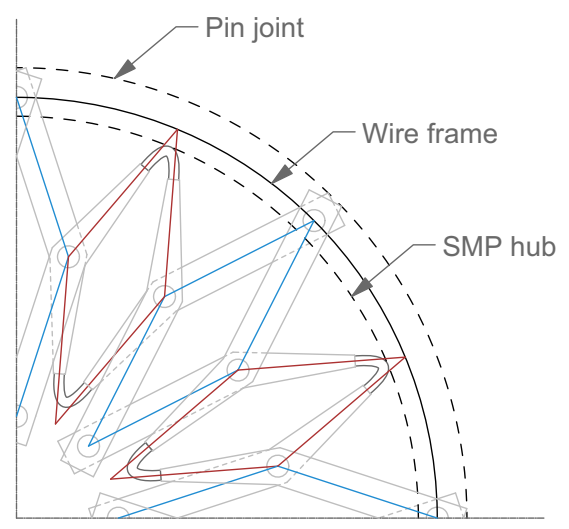

FIG. 12. If the arm lengths of the scissors are uniform, the physical outer radii will differ depending on whether the end of the scissor mechanism is a SMP hub or a pin joint. The variable $\xi$ is introduced to ensure that these two radii are identical.

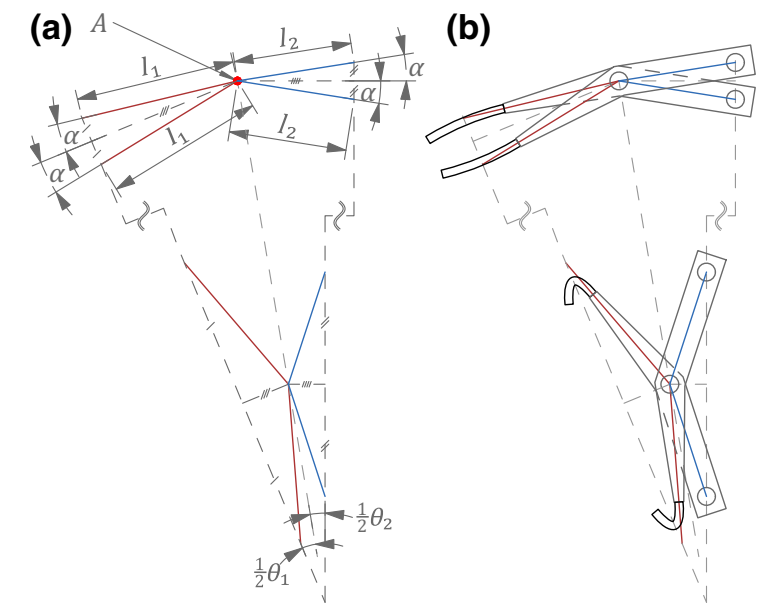

FIG. 13. A detailed analysis of the collapse motion of one scissor mechanism, with and without the outlining physical geometry.

We propose to vary the lengths of $e_{1}$ and $e_{2}$ by moving the position of point $A$ along the edge by multiplying the parameter $t$ by a factor $\xi$. This factor is bounded by $\left(0, s^{*} / s\right)$. Polygon II is consequently turned into an isogonal polygon. Specifically, the lengths $s_{1}$ and $s_{2}$ can be derived as follows:

$$
s_{1}=\xi t s^{*}, \quad s_{2}=\cos (\gamma)(1-\xi t) s^{*} .
$$

It can be seen that the collapsing motion of the scissor mechanisms is exactly bounded by a wedge shape (Fig. 13). The angles of this wedge can be derived as follows:

$$
\theta_{1}=2 \arctan \left(\frac{s_{1}}{2 r_{\mathrm{IC}, \mathrm{I}}}\right), \quad \theta_{2}=2 \gamma-\theta_{1} .
$$

The collapse motion can be parametrized by the half angle between two arms of the scissor mechanism, $\alpha$. We prescribe an initial angle $\alpha=\alpha_{\min }$ of the scissor mechanisms and derive the lengths $l_{1}$ and $l_{2}$. Note that each scissor mechanism has a length of $l_{1}+l_{2}$. These lengths can be derived as follows:

$$
l_{1}=\frac{1}{2} \frac{s_{1}}{\cos \left(\alpha_{\min }\right)}, \quad l_{2}=\frac{1}{2} \frac{s_{2}}{\cos \left(\alpha_{\min }\right)} .
$$

Assuming that the width of each scissor, $w$, equals the edge length of a fictitious inner polygon of edge $2 n$, called polygon III (Fig. 14), the inscribing radius $r_{\mathrm{IC}, \mathrm{III}}$ is then as follows:

$$
r_{\mathrm{IC}, \mathrm{III}}=\frac{1}{2} w \cot (\gamma / 2)
$$




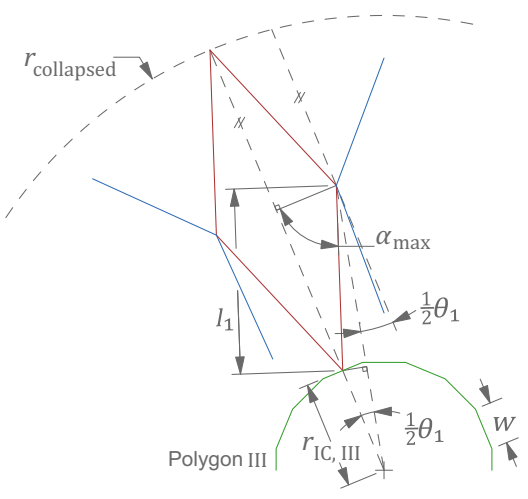

FIG. 14. The fully collapsed configuration, showing the variables needed to derived the collapsed outer radius.

We now derive the maximum collapse angle $\alpha_{\max }$ considering the physical dimensions. Considering trigonometry (Fig. 14), the maximum angle of rotation $\alpha_{\max , 1}$ of scissor $l_{1}$ and similarly for $l_{2}$ is therefore as follows:

$$
\begin{aligned}
& \alpha_{\mathrm{max}, 1}=\frac{\pi}{2}-\frac{\theta_{1}}{2}-\arcsin \frac{r_{\mathrm{IC}, \mathrm{III}} \sin \left(\theta_{1} / 2\right)}{l_{1}}, \\
& \alpha_{\max , 2}=\frac{\pi}{2}-\frac{\theta_{1}}{2}-\arcsin \frac{r_{\mathrm{IC}, \mathrm{III}} \sin \left(\theta_{2} / 2\right)}{l_{2}} .
\end{aligned}
$$

Consider the following simplifications:

$$
\begin{gathered}
\theta_{1}+\theta_{2}=\gamma, \\
\sin \left(\theta_{2}\right)=\frac{l_{2}}{l_{1}} \sin \left(\theta_{1}\right) .
\end{gathered}
$$

The mean value of the two angles can be obtained as follows:

$$
\begin{gathered}
\alpha_{\max }=\frac{1}{2}\left(\alpha_{\max , 1}+\alpha_{\max , 2}\right) \\
=\frac{\gamma(n-1)}{2}-\arcsin \frac{r_{\mathrm{IC}, \mathrm{III}} \sin \left(\theta_{1} / 2\right)}{l_{1}} .
\end{gathered}
$$

Similarly, the collapsed radius $r_{\min }$ can be derived as follows:

$$
\begin{gathered}
r_{\min }=\frac{1}{2}\left(r_{\min , 1}+r_{\min , 2}\right) \\
=r_{\mathrm{IC}, \mathrm{III}}+\left(l_{1}+l_{2}\right) \sin \left(\alpha_{\max }\right) .
\end{gathered}
$$

The expanded radius $r_{\max }$ refers to polygon I from the start of the derivation:

$$
r_{\max }=r_{\mathrm{IC}, \mathrm{I}}+\left(l_{1}+l_{2}\right) \sin \left(\alpha_{\min }\right) .
$$

We define the ratio of area expansion as follows:

$$
\Delta A=\left(\frac{r_{\max }}{r_{\min }}\right)^{2}
$$

In Fig. 2, we plot $\Delta A$ vs $n$ for different values of $w$. The explicit form of $\Delta A$ is omitted for brevity.

\section{APPENDIX B: OPTIMIZATION LANDSCAPE AND CONSTRAINT BOUNDARIES OF THE "FLASHER" ORIGAMI}

In this work, we adopt the "flasher" origami as the basis for attachment of the solar panels. We relate the shape and folds of a flash origami pattern to the number of solar panels that can fit onto such a pattern. With this, we are able to generate a crease pattern that can accommodate the given number of solar panels of a certain size. We also use this derivation to relate the radius of the unfolded pattern to the folded one.

Given two concentric polygons of $n$ sides, we rotate the outer polygon such that the edges of the inner polygon, if extended outward, bisect the edges of the outer polygon [Fig. 15(a), "Line 1"]. These two polygons define the outer and inner limits of the unfolded pattern. Two additional polygons of $n$ sides are similarly used to define the outer and the inner limits of the folded pattern. These four polygons are denoted with subscripts $u, o, u, i, f, o$, and ${ }_{f, i}$, referring to unfolded outer, folded outer, unfolded inner, and folded inner, respectively, e.g., the circumscribing radius, the inscribing radius, and the edge length of the unfolded outer polygon are denoted as $R_{u, o}, r_{u, o}$, and $s_{u, o}$, respectively. The superscript ${ }^{*}$ refers to quantities offset by the aforementioned rotation of the two outer polygons [Fig. 15(a)].

The known quantities are $R_{u, o}{ }^{*}, R_{f, i}, R_{u, i}$, as they are determined by the maximum volume and minimum resolution of the 3D printer. We define $\gamma=\pi / n$.

We first list the relations for later derivation. The circumscribing and the inscribed radius of the unfolded outer polygon, as well as the inscribed radius of the unfolded 

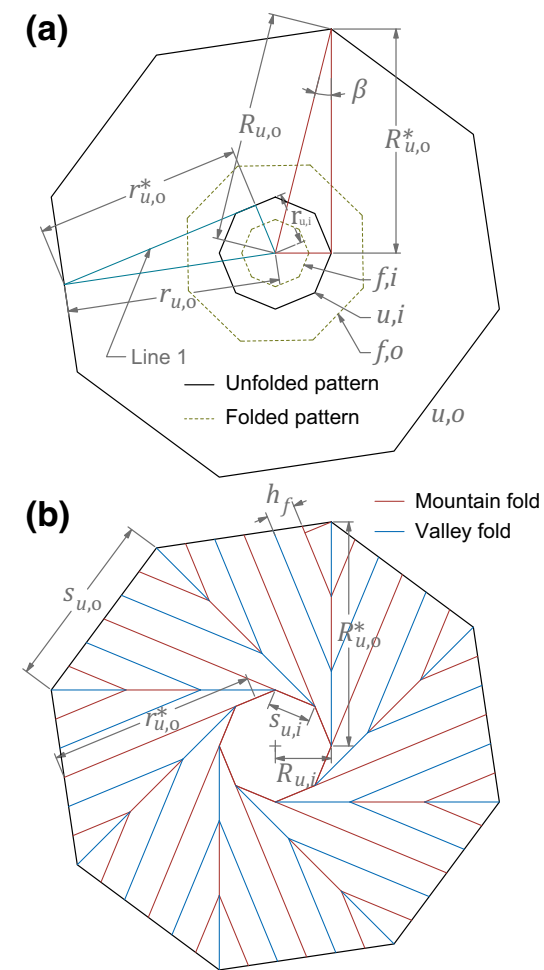

FIG. 15. The construction of a crease pattern of a "flasher" origami. The variables used in the derivation are shown.

inner polygon, are as follows:

$$
\begin{array}{ll}
R_{u, o}=\left(R_{u, o}{ }^{* 2}+R_{u, i}^{2}\right)^{1 / 2}, & r_{u, o}{ }^{*}=\left(r_{u, o}{ }^{2}-r_{u, i}{ }^{2}\right)^{1 / 2}, \\
r_{u, o}=R_{u, o} \cos (\gamma), & r_{u, i}=R_{u, i} \cos (\gamma) .
\end{array}
$$

The rotation between the outer and the inner polygon is defined as the following:

$$
\beta=\arctan \left(\frac{r_{u, i}}{R_{u, o}{ }^{*}}\right) .
$$

We calculate the edge lengths:

$$
\begin{gathered}
s_{u, o}=2 R_{u, o} \sin (\gamma), \quad s_{f, i}=2 R_{f, i} \sin (\gamma), \\
s_{u, i}=2 R_{u, i} \sin (\gamma) .
\end{gathered}
$$

The enveloping outer radius of the folded geometry is defined in relation to the inner radius of the folded geometry and the number of layers $p$ wrapped around the center. The variables $c$ and $t_{f}$ refer to the number of levels in the "flasher" pattern and the thickness of the origami facets, respectively. The second term on the right-hand side of Eq.
(B6) calculates the maximum thickness possible given the number of "flasher" layers and is derived by counting the number of mountain folds in any radial cross section:

$$
R_{f, o}=R_{f, i}+2 t_{f} p c
$$

The number of edges that each arm of the outer polygon can wrap around the inner polygon is calculated as the ratio between the inscribed radius of the unfolded geometry $r_{u, o}{ }^{*}$ and the edge length of the folded geometry, $s_{f, o}$. Note that this edge length increases as more layers are wrapped and $r_{f, o}$ increases,

$$
p=\frac{r_{u, o}{ }^{*}-s_{u, i} / 2}{s_{f, o}}
$$

and the edge length of the folded polygon follows:

$$
s_{f, o}=2 R_{f, o} \sin (\gamma)
$$

We solve for $s_{f, o}$ noting that the above is an implicit equation, as $R_{f o}$ depends on $p$, which, in turn, is a function of $s_{f, o}$ :

$$
\begin{aligned}
s_{f, o}= & R_{f, i} \sin (\gamma)+\left\{\operatorname { s i n } ( \gamma ) \left[-4 \sin (\gamma) R_{u, i} c t\right.\right. \\
& \left.\left.+\sin (\gamma) R_{f, i}^{2}+4 \cos (\gamma) R_{u, o}{ }^{*} c t\right]\right\} .
\end{aligned}
$$

Once $s_{f, o}$ is obtained, $R_{f, o}$ is derived from Eq. (B8) and forms the $g_{2}$ constraint in Fig. 2.

The $g_{1}$ constraint requires the height of the folded origami $h_{f}$ to be greater than the smaller dimension of the solar panels. This allows the PV cells to be placed on the facets and not on the crease lines. The height simply relates to the edge length of the unfolded polygon divided by twice the number of layers $2 c$ in the "flasher" pattern:

$$
h_{f}=\frac{s_{u, o}}{2 c} \cos (\beta) .
$$

Lastly, the optimization problem [Eq. (B11)] is derived. We frame the problem by assuming one type of rectangular flexible PV cell $\left(l_{\mathrm{LV}} \times w_{\mathrm{PV}}\right)$ that can only be placed on the facets of the origami pattern [e.g., Fig. 15(c)]. We would like to optimize the crease pattern by varying both $n$ and $c$ to maximize the number of PV cells that are able to fit:

$$
\underset{c, n}{\operatorname{minimize}} \quad-\max [p(c, n), 0]
$$

The number of PV cells that can be placed upon the origami must be a discrete number and is derived by considering one radial unit cell of the pattern (Fig. 16). Due to the asymmetry of the pattern, the + and - directions are 
considered separately. The result is similarly shown in two parts [see Eq. (B13)]:

$$
\begin{aligned}
p(c, n)= & n \sum_{i=1}^{c} \text { floor }\left\{\frac { 1 } { l _ { \mathrm { PV } } } \left[r_{u, o}{ }^{*}+\frac{1}{2} s_{u, i}-w_{\mathrm{PV}} \cot (\gamma)\right.\right. \\
& -(i-1) h_{f} \cot (\gamma)-w_{\mathrm{PV}} \tan (\beta) \\
& \left.\left.-(i-1) h_{f} \tan (\beta)\right]\right\} \\
& +n \sum_{i=1}^{c} \text { floor }\left\{\frac { 1 } { l _ { \mathrm { PV } } } \left[r_{u, o}{ }^{*}-\frac{1}{2} s_{u, i}-w_{\mathrm{PV}} \cot (\gamma)\right.\right. \\
& \left.\left.-(i-1) h_{f} \cot (\gamma)+(i-1) h_{f} \tan (\beta)\right]\right\}, \\
p(c, n)= & n \sum_{i=1}^{c} \text { floor }\left(\frac { 1 } { l _ { \mathrm { PV } } } \left\{r_{u, o}{ }^{*}+\frac{1}{2} s_{u, i}\right.\right. \\
& \left.\left.-\left[w_{\mathrm{PV}}+(i-1) h_{f}\right][\cot (\gamma)+\tan (\beta)]\right\}\right) \\
& +n \sum_{i=1}^{c} \text { floor }\left(\frac { 1 } { l _ { \mathrm { PV } } } \left\{r_{u, o}{ }^{*}-\frac{1}{2} s_{u, i}\right.\right. \\
& -\left[w_{\mathrm{PV}}+(i-1) h_{f}\right] \cot (\gamma) \\
& \left.\left.+(i-1) h_{f} \tan (\beta)\right\}\right) . \\
& (\mathrm{B} 13) \\
&
\end{aligned}
$$

The two constraints $\left(g_{1}\right.$ and $\left.g_{2}\right)$ previously derived are stated below to complete the problem statement:

$$
\begin{gathered}
g_{1}: w_{\mathrm{PV}}-h_{f}+2 t_{c} \leq 0 . \\
g_{2}: r_{f, o}-r_{\mathrm{IC}, \mathrm{III}} \leq 0 .
\end{gathered}
$$

\section{APPENDIX C: COLLAPSING PROCEDURE}

Thermomechanically, the procedure for collapsing the system from the deployed configuration to the stowed configuration is referred to as "programming." It is done at a temperature higher than the glass-transition temperature of the SMPs within the system.

The components of the system (i.e., the "flasher" origami, the scissor mechanisms, and the connections between the two) are fabricated in the deployed configuration and assembled along with the solar panels [Figs. 17(a) and $17(\mathrm{c})]$. A center core is installed and connected to the "flasher" origami through a number of flexible wires [Figs. 17(b) and 17(d)].

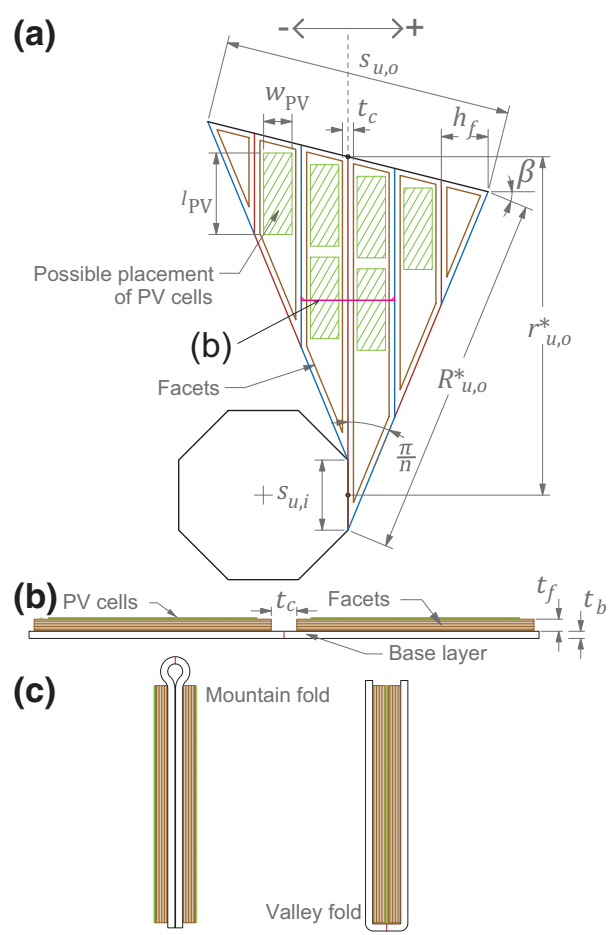

FIG. 16. One unit cell of the origami pattern, with variables used in the derivation of the optimization objective indicated. The details of the mountain and valley folds are shown in both the flat and the folded configurations.

The assembled system is submerged in heated water $\left(T \geq T_{g}\right)$, and the center core is rotated as the crease lines are folded. This continues until the collapsed system fits within a prescribed cylinder [Fig. 17(e)]. Once in the cylinder and cooled to below the glass-transition temperature, the cylinder is released [Fig. 17(f)].

\section{APPENDIX D: MATERIAL CHARACTERIZATION AND SPECIMEN FABRICATION}

The solar-panel specimen is fabricated entirely using a commercial 3D printer, the Stratasys Connex3 Objet500. Two materials are used, FLX9895 and RGD835. FLX9895 is the SMP used to fabricate both the origami substrate and the hubs in the scissor mechanisms. RGD835 is intended to be stiff during deployment and after.

Both FLX9895 and RGD835 are characterized using DSC to determine their glass-transition temperature. The results show that the $T_{g}$ values of FLX9895 and RGD835 are $35{ }^{\circ} \mathrm{C}$ and $65^{\circ} \mathrm{C}$, respectively. With an intended activation temperature of $35{ }^{\circ} \mathrm{C}$, RGD835 is guaranteed to remain stiff during and after deployment.

For the SMP, the fully relaxed modulus and frequency sweeps of the storage modulus at different temperatures are obtained. The fully relaxed modulus is obtained using an Instron E3000 dynamic testing machine with a $3 \mathrm{kN}$ load cell and a temperature-controlled chamber. 

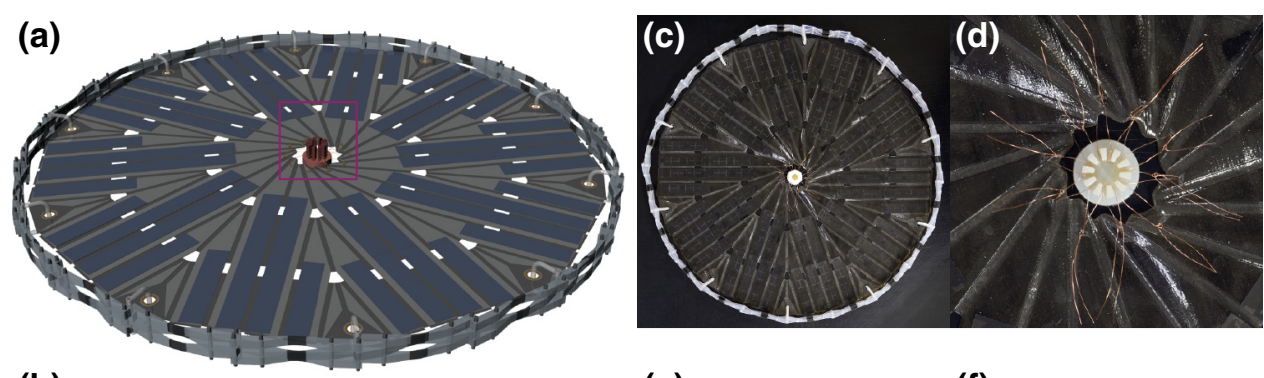

(b)

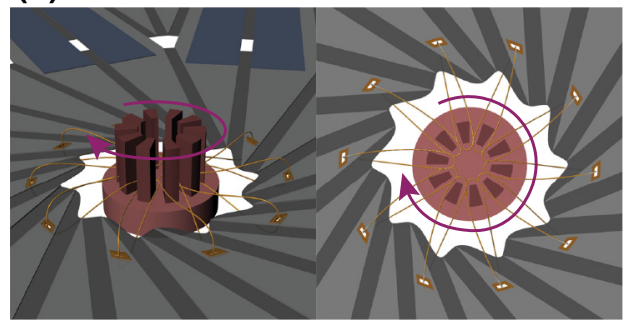

(e)

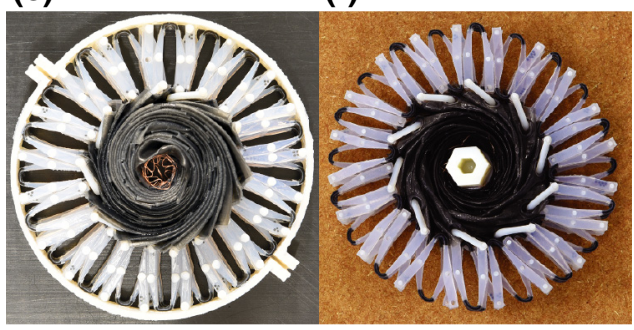

FIG. 17. The "programming" of the solar-panel array. (a),(c) The procedure starts with the deployed configuration. (b),(d) A center rotational core is installed and connected with the origami via flexible wires. (e) In a heated environment, the system is rotated and folded until it fits within a cylindrical mold. (f) After cooling within the mold, it is released and stable on its own.

TABLE I. Prony series of FLX9895 as implemented in ABAQUS.

\begin{tabular}{rr}
\hline \hline & FLX9895 \\
\hline $\boldsymbol{\tau}_{\boldsymbol{i}}$ & $\boldsymbol{G}_{\boldsymbol{i}}$ \\
\hline $2.00 \times 10^{-9}$ & 0.037155 \\
$1.00 \times 10^{-8}$ & 0.036325 \\
$5.00 \times 10^{-8}$ & 0.019428 \\
$1.00 \times 10^{-7}$ & 0.036963 \\
$5.00 \times 10^{-7}$ & 0.025824 \\
0.000001 & 0.048254 \\
0.000005 & 0.029066 \\
0.00001 & 0.061186 \\
0.00005 & 0.047911 \\
0.0001 & 0.048443 \\
0.0005 & 0.046758 \\
0.001 & 0.081033 \\
0.005 & 0.088524 \\
0.01 & 0.069187 \\
0.05 & 0.11156 \\
0.1 & 0.043557 \\
0.5 & 0.079339 \\
1 & 0.023205 \\
5 & 0.038059 \\
10 & 0.004878 \\
50 & 0.015653 \\
500 & 0.00000 \\
1000 & 0.003613 \\
5000 & 0.000806 \\
5000000000 & 0.001064 \\
1000000 & 0.000212 \\
\hline \hline & 0.000218 \\
\hline & 0.000239 \\
\hline & \\
\hline &
\end{tabular}

The experiments are performed at $60{ }^{\circ} \mathrm{C}$ with a rate of $0.05 \mathrm{~mm} \mathrm{~s}^{-1}$. Cylindrical specimens with a diameter of $7 \mathrm{~mm}$ and a height of $9 \mathrm{~mm}$ are used. The storage modulus is obtained using a dynamic-mechanical-analysis (DMA) (Mettler Toledo DMA 861) machine. Specimens $(3.00 \times$ $4.30 \times 2.05 \mathrm{~mm}^{3}$ ) are tested under oscillatory shear (frequency $0.01-100 \mathrm{~Hz}$, amplitude $0.5 \mu \mathrm{m}$ ) at temperatures of $-40,-17,2,12,21,30,40$, and $49^{\circ} \mathrm{C}$.

We assume that the SMP is thermologically simple and follows the time-temperature superposition principle, which assumes that the behavior of the polymer at a given temperature can be approximated as a product of the relaxation times at a reference temperature $T_{\text {ref }}$ and the shift factors $a_{T}$ at that temperature [Eq. (D1)]:

$$
\tau_{i}(T)=a_{T} \tau_{i}\left(T_{\text {ref }}\right) .
$$

These shift factors as approximated by the WilliamsLandel-Ferry (WLF) equation [Eq. (D2)] are used to construct a master curve of the storage modulus applicable at a reference temperature, in this case $307.15 \mathrm{~K}$ [39]. This is set to coincide with the $T_{g}$ value of the SMP:

$$
\log _{10} a_{T}=\log _{10} \frac{\tau(T)}{\tau\left(T_{\text {ref }}\right)}=-\frac{C_{1}\left(T-T_{\text {ref }}\right)}{C_{2}+\left(T-T_{\text {ref }}\right)}, \quad T>T_{\text {ref }} .
$$

The finite-element package, ABAQUS, requires the relaxation modulus in the time domain as an input. A Fourier transform is then used for the translation [Eq. (D3)] [39]:

$$
G(t)=G_{\infty}+\frac{2}{\pi} \int_{0}^{\infty}\left[\frac{G^{\prime}-G_{\infty}}{\omega}\right] \sin \omega t d \omega
$$


TABLE II. TRS coefficients as input in ABAQUS for FLX9895.

\begin{tabular}{ll}
\hline & FLX9895 \\
\hline$T_{\text {ref }}$ & 275.00 \\
\hline$C_{1}$ & 19.987 \\
$C_{2}$ & 109.53 \\
\hline \hline
\end{tabular}

This Fourier transform [Eq. (D3)] is approximated discretely and evaluated numerically [40]. Eleven terms are used to obtain a sufficiently accurate approximation. Using the fitted coefficient, a Prony series [Eq. (D4)] of the SMP with 29 terms is constructed (Tables I and II):

$$
G(t)=G_{\infty}+\sum_{i=1}^{N} G_{i} e^{-t / \tau_{i}} .
$$

[1] James O. McSpadden and John C. Mankins, Space solar power programs and microwave wireless power transmission technology, IEEE Microw. Mag. 3, 46 (2002).

[2] Thomas McGuire, Michael Hirsch, Michael Parsons, Skye Leake, and Jeremy Straub, A CubeSat deployable solar panel system, Proc. SPIE 9865, 9865 (2016).

[3] Deborah Sigel, Brian P. Trease, Mark W. Thomson, David R. Webb, Paul Willis, and P. Lisman, in Proceedings of IDETC/CIE (Buffalo, New York, 2014), p. DETC2014.

[4] Yiqun Zhang, Dongwu Yang, and Sunlan Li, An integrated control and structural design approach for mesh reflector deployable space antennas, Mechatronics 35, 71 (2014).

[5] Osamu Mori, Hirotaka Sawada, Ryu Funase, Mutsuko Morimoto, Tatsuya Endo, Takayuki Yamamoto, Yuichi Tsuda, Yasuhiro Kawakatsu, and Yoji Shirasawa, First solar power sail demonstration by IKAROS, Trans. JSASS 8, To_4_25 (2010).

[6] M. Schenk, S. G. Kerr, A. M. Smyth, and Simon D. Guest, in Proceedings of the First Conference Transformables, September, edited by Felix Escrig and Jose Sanchez (Starbooks, School of Architecture, Seville, Spain, 2013), p. 18.

[7] Joachim Block, Marco Straubel, and Martin Wiedemann, Ultralight deployable booms for solar sails and other large gossamer structures in space, Acta Astronaut. 68, 984 (2011).

[8] Elliot Hawkes, B. An, Nadia M. Benbernou, H. Tanaka, Sangbae Kim, E. D. Demaine, D. Rus, and Robert J. Wood, Programmable matter by folding, Proc. Natl. Acad. Sci. 107, 12441 (2010).

[9] Zi Chen, Gaoshan Huang, Ian Trase, Xiaomin Han, and Yongfeng Mei, Mechanical Self-Assembly of a StrainEngineered Flexible Layer: Wrinkling, Rolling, and Twisting, Phys. Rev. Appl. 5, 017001 (2016).

[10] Skylar Tibbits, 4D printing: Multi-material shape change, Arch. Des. 84, 116 (2014).

[11] Dan Raviv, Wei Zhao, Carrie McKnelly, Athina Papadopoulou, Achuta Kadambi, Boxin Shi, Shai Hirsch, Daniel Dikovsky, Michael Zyracki, Carlos Olguin, Ramesh Raskar, and Skylar Tibbits, Active printed materials for complex self-evolving deformations, Sci. Rep. 4, 7422 (2014).

[12] Yiqi Mao, Kai Yu, Michael S. Isakov, Jiangtao Wu, Martin L. Dunn, and H. Jerry Qi, Sequential self-folding structures by $3 \mathrm{D}$ printed digital shape memory polymers, Sci. Rep. $\mathbf{5}$, 13616 (2015).

[13] Marius Wagner, Tian Chen, and Kristina Shea, Large shape transforming 4D auxetic structures, 3D Print. Addit. Manuf. 4, 133 (2017).

[14] Timothy J. White and Dirk J. Broer, Programmable and adaptive mechanics with liquid crystal polymer networks and elastomers, Nat. Mater. 14, 1087 (2015).

[15] Anton A. Bauhofer, Sebastian Krödel, Jan Rys, Osama R. Bilal, Andrei Constantinescu, and Chiara Daraio, Harnessing photochemical shrinkage in direct laser writing for shape morphing of polymer sheets, Adv. Mater. 29, 1703024 (2017).

[16] Robert F. Shepherd, Filip Ilievski, Wonjae Choi, Stephen A. Morin, Adam A. Stokes, Aaron D. Mazzeo, Xin Chen, Michael Wang, and George M. Whitesides, Multigait soft robot, Proc. Natl. Acad. Sci. 108, 20400 (2011).

[17] Michael Wehner, Ryan L. Truby, Daniel J. Fitzgerald, Bobak Mosadegh, George M. Whitesides, Jennifer A. Lewis, and Robert J. Wood, An integrated design and fabrication strategy for entirely soft, autonomous robots, Nature 536, 451 (2016).

[18] J. H. Pikul, S. Li, H. Bai, R. T. Hanlon, I. Cohen, and R. F. Shepherd, Stretchable surfaces with programmable 3D texture morphing for synthetic camouflaging skins, Science 358, 210 (2017).

[19] Tian Chen and Kristina Shea, An autonomous programmable actuator and shape reconfigurable structures using bistability and shape memory polymers, 3D Print. Addit. Manuf. 5, 91 (2018).

[20] Tian Chen, Osama R. Bilal, Kristina Shea, and Chiara Daraio, Harnessing bistability for directional propulsion of soft, untethered robots, Proc. Natl. Acad. Sci. 115, 5698 (2018).

[21] Ryan L. Truby and Jennifer A. Lewis, Printing soft matter in three dimensions, Nature 540, 371 (2016).

[22] Charles Hoberman, Reversibly expandable doubly curved truss structure, U.S. Patent 4,942,700 (1990).

[23] Jeremy Shafer, Origami to Astonish and Amuse (St. Martin's Griffin, Manhattan, New York City, 2001), 1st ed., p. 1.

[24] Charles Hoberman, Folding covering panels for expanding structures, U.S. Patent 6,834,465 B2 (2004).

[25] Charles L. Larson, Scissor-Lift Mechanism, U.S. Patent 3,246,876 (1966).

[26] Zhong You and Sergio Pellegrino, Foldable bar structures, Int. J. Solids Struct. 34, 1825 (1997).

[27] Jiten Patel and G. K. Ananthasuresh, A kinematic theory for radially foldable planar linkages, Int. J. Solids Struct. 44, 6279 (2007).

[28] Koryo Miura, Method of packaging and deployment of large membranes in space, Inst. Space Astronaut. Sci. Rep. 618, 1 (1985).

[29] Tomohiro Tachi, Simulation of rigid origami, Origami 4, 175 (2009).

[30] Qi Ge, Conner K. Dunn, H. Jerry Qi, and Martin L. Dunn, Active origami by 4D printing, Smart Mater. Struct. 23, 094007 (2014). 
[31] Shannon A. Zirbel, Robert J. Lang, Mark W. Thomson, Deborah A. Sigel, Phillip E. Walkemeyer, Brian P. Trease, Spencer P. Magleby, and Larry L. Howell, Accommodating thickness in origami-based deployable arrays, J. Mech. Des. 135, 111005 (2013).

[32] Robert J. Lang, Spencer Magleby, and Larry Howell, Single degree-of-freedom rigidly foldable cut origami flashers, J. Mech. Robot. 8, 031005 (2015).

[33] Katia Bertoldi, Vincenzo Vitelli, Johan Christensen, and Martin van Hecke, Flexible mechanical metamaterials, Nat. Rev. Mater. 2, 17066 (2017).

[34] Shahram Janbaz, Molly McGuinness, and Amir A. Zadpoor, Multimaterial Control of Instability in Soft Mechanical Metamaterials, Phys. Rev. Appl. 9, 064013 (2018)
[35] Tian Chen, Jochen Mueller, and Kristina Shea, Integrated design and simulation of tunable, multi-state structures fabricated monolithically with multi-material 3D printing, Sci. Rep. 7, 45671 (2017).

[36] W. E. Lanford, Folding Apparatus, U.S. Patent 3,010,372 (1961)

[37] Mark Schenk and Simon D. Guest, in Origami 5: Fifth International Meeting of Origami Science, Mathematics, and Education (CRC Press, Boca Raton), p. 291.

[38] Amanda Ghassaei, Origami simulator (2017).

[39] JohnD. Ferry, Viscoelastic Properties of Polymers (John Wiley \& Sons, Inc., Toronto, 1980), 3rd ed., p. 1.

[40] F. R. Schwarzl, Numerical calculation of stress relaxation modulus from dynamic data for linear viscoelastic materials, Rheol. Acta. 14, 581 (1975). 